\title{
Unveiling Shrouded Oceans on Temperate sub-Neptunes via Transit Signatures of Solubility Equilibria versus Gas Thermochemistry
}

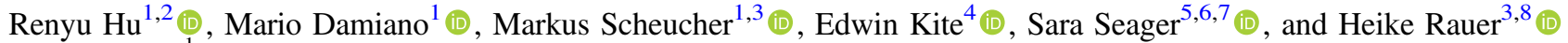 \\ ${ }^{1}$ Jet Propulsion Laboratory, California Institute of Technology, Pasadena, CA 91109, USA; renyu.hu@jpl.nasa.gov \\ ${ }^{2}$ Division of Geological and Planetary Sciences, California Institute of Technology, Pasadena, CA 91125, USA \\ ${ }^{3}$ Institut für Planetenforschung, Deutsches Zentrum für Luft- und Raumfahrt, D-12489 Berlin, Germany \\ ${ }^{4}$ Department of the Geophysical Sciences, University of Chicago, Chicago, IL 60637, USA \\ ${ }^{5}$ Department of Earth, Atmospheric, and Planetary Sciences, Massachusetts Institute of Technology, Cambridge, MA 02139, USA \\ ${ }^{6}$ Department of Physics and Kavli Institute for Astrophysics and Space Research, Massachusetts Institute of Technology, Cambridge, MA 02139, USA \\ 7 Department of Aeronautics and Astronautics, Massachusetts Institute of Technology, Cambridge, MA 02139, USA \\ ${ }^{8}$ Institut für Geologische Wissenschaften, Freie Universität Berlin, D-12249 Berlin, Germany \\ Received 2021 July 28; revised 2021 August 10; accepted 2021 August 15; published 2021 October 28
}

\begin{abstract}
The recent discovery and initial characterization of sub-Neptune-sized exoplanets that receive stellar irradiance of approximately Earth's raised the prospect of finding habitable planets in the coming decade, because some of these temperate planets may support liquid-water oceans if they do not have massive $\mathrm{H}_{2} / \mathrm{He}$ envelopes and are thus not too hot at the bottom of the envelopes. For planets larger than Earth, and especially planets in the 1.7-3.5 $R_{\oplus}$ population, the mass of the $\mathrm{H}_{2} / \mathrm{He}$ envelope is typically not sufficiently constrained to assess the potential habitability. Here we show that the solubility equilibria versus thermochemistry of carbon and nitrogen gases typically results in observable discriminators between small $\mathrm{H}_{2}$ atmospheres versus massive ones, because the condition to form a liquid-water ocean and that to achieve the thermochemical equilibrium are mutually exclusive. The dominant carbon and nitrogen gases are typically $\mathrm{CH}_{4}$ and $\mathrm{NH}_{3}$ due to thermochemical recycling in a massive atmosphere of a temperate planet, and those in a small atmosphere overlying a liquid-water ocean are most likely $\mathrm{CO}_{2}$ and $\mathrm{N}_{2}$, followed by $\mathrm{CO}$ and $\mathrm{CH}_{4}$ produced photochemically. $\mathrm{NH}_{3}$ is depleted in the small atmosphere by dissolution into the liquid-water ocean. These gases lead to distinctive features in the planet's transmission spectrum, and a moderate number of transit observations with the James Webb Space Telescope should tell apart a small atmosphere versus a massive one on planets like K2-18 b. This framework thus points to a way to use nearterm facilities to constrain the atmospheric mass and habitability of temperate sub-Neptune exoplanets.
\end{abstract}

Unified Astronomy Thesaurus concepts: Exoplanet atmospheres (487); Extrasolar rocky planets (511); Extrasolar ice giants (2024); Habitable planets (695); Ocean planets (1151); Transmission spectroscopy (2133)

\section{Introduction}

The exoplanet community already has ways to detect an $\mathrm{H}_{2}$ atmosphere by transmission spectroscopy via its pressure scale height 1 order of magnitude larger than that of an $\mathrm{N}_{2}$ or $\mathrm{CO}_{2}$ atmosphere (Miller-Ricci et al. 2008). However, the mass of the $\mathrm{H}_{2}$ atmosphere- the parameter that controls the temperature at the bottom of the atmosphere and thus the possibility for liquid water (Pierrehumbert \& Gaidos 2011; Ramirez \& Kaltenegger 2017; Koll \& Cronin 2019) - is not directly measurable from the transmission spectrum. Also, a planet's mass and radius typically allow multiple models of the interior structure (e.g., Rogers \& Seager 2010; Valencia et al. 2013). It is unclear whether the planets in the 1.7-3.5 $R_{\oplus}$ population (Fulton \& Petigura 2018) are mostly rocky planets with massive $\mathrm{H}_{2} / \mathrm{He}$ gas envelopes (Owen \& Wu 2017; Jin \& Mordasini 2018) or planets with a massive water layer $(\sim 50 \mathrm{wt} \%)$ that do not require a large $\mathrm{H}_{2}$ envelope to explain their radius (e.g., referred to as "ocean planets" hereafter; Zeng et al. 2019; Mousis et al. 2020; Venturini et al. 2020). Direct-imaging observations in the future may provide means to detect a surface underneath a thin atmosphere on temperate planets, via the ocean glint (Robinson et al. 2010) or surface heterogeneity (Cowan et al. 2009; Fan et al. 2019). However, these methods are not applicable to the near-term capabilities such as the James Webb Space Telescope (JWST) and may pose challenges on precision even for ambitious direct-imaging mission concepts (Gaudi et al. 2020).
The temperate sub-Neptune K2-18 b is a harbinger of the class of planets that might be habitable and exemplifies the need for a near-term method to measure the size of an $\mathrm{H}_{2}$ atmosphere. The planet of 8.6 $M_{\oplus}$ and $2.6 R_{\oplus}$ is in the habitable zone of an $\mathrm{M}$ dwarf star, and has a transmission spectrum (obtained by Hubble at 1.1-1.7 $\mu \mathrm{m}$ ) with confirmed spectral features, which indicates that the planet should host an atmosphere dominated by $\mathrm{H}_{2}$ (Benneke et al. 2019; Tsiaras et al. 2019). Interior structure models showed that the planet can have a massive ( $>\sim 1000$ bar) $\mathrm{H}_{2}$ atmosphere overlaying a rocky $/ \mathrm{Fe}$ core and a possibly supercritical water layer, or a smaller $\left(<100\right.$ bar) $\mathrm{H}_{2}$ atmosphere with a water-dominated interior (Madhusudhan et al. 2020; Mousis et al. 2020; Nixon \& Madhusudhan 2021). For K2-18 b, specifically, a $\sim 10-100$ bar $\mathrm{H}_{2}$ atmosphere overlaying a water layer would cause $>200$ bar of water to evaporate into the atmosphere, resulting in a hot steam atmosphere inconsistent with the observed transmission spectrum (Scheucher et al. 2020). An even smaller, $\sim 1$ bar $\mathrm{H}_{2}$ atmosphere would prevent this steam atmosphere and produce a liquid-water ocean (see Section 3), but this requires a very small rocky/Fe core and may be disfavored from the planet formation standpoint (e.g., Lee \& Chiang 2016). However, a planet slightly more massive or smaller than $\mathrm{K} 2-18 \mathrm{~b}$ - such as those at the center of the 1.7-3.5 $R_{\oplus}$ planet population-does not have this small-core difficulty to have a small atmosphere (Zeng et al. 2019; Nixon \& Madhusudhan 2021), and many such planets and planet candidates have been detected and will soon be available for transmission spectroscopy (Figure 1, panel (a)). 


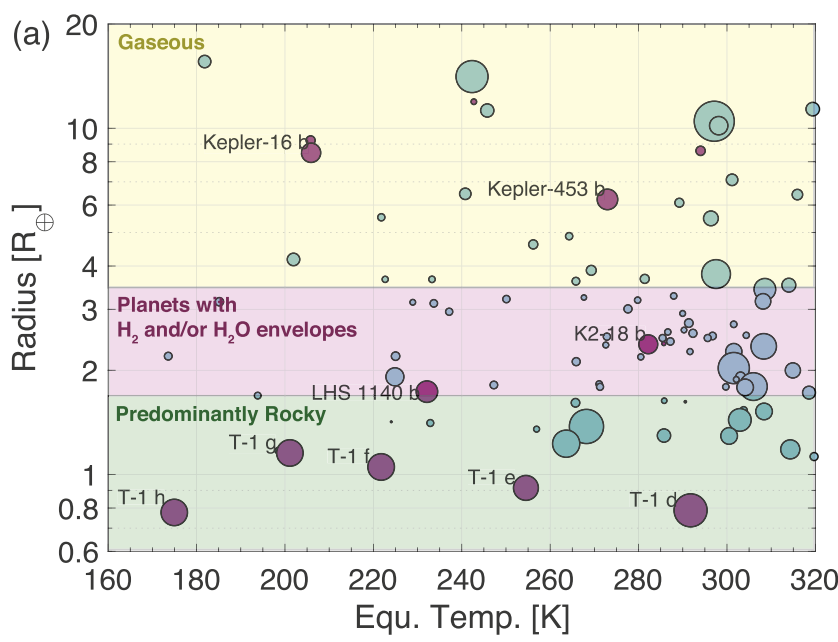

(b)

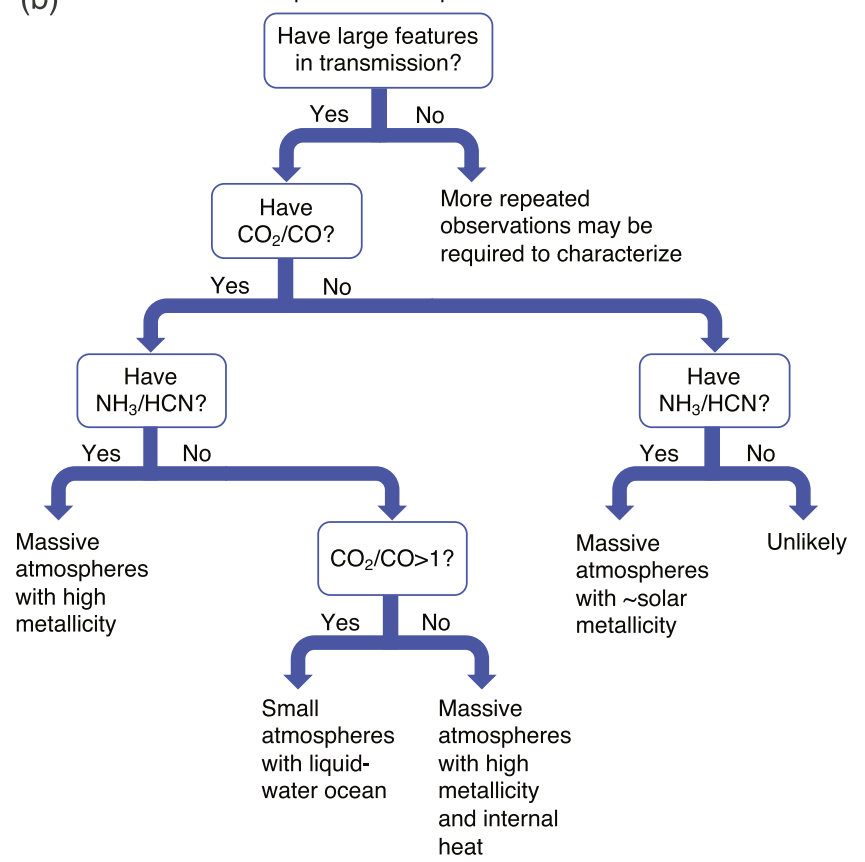

Figure 1. Temperate exoplanets amenable for atmospheric characterization via transmission spectroscopy. (a) Purple dots are confirmed planets with measured masses, and blue dots are planets with unknown masses or planet candidates. Data are taken from the NASA Exoplanet Archive and and the TESS Objects of Interest Catalog. The marker sizes are scaled with the expected signal-tonoise ratio of the spectral features of an $\mathrm{H}_{2}$ atmosphere observed by JWST at $2 \mu \mathrm{m}$. Most of the temperate planets and planet candidates suitable for atmospheric characterization are larger than Earth and thus more likely to have $\mathrm{H}_{2}$ atmospheres. (b) A roadmap to characterize the mass of the atmospheres and the habitability of temperate sub-Neptunes by detecting signature gases. See the text for details.

Here we propose that transit observations of temperate subNeptunes in the near- and mid-infrared wavelengths, which will soon commence with JWST, can detect small $\mathrm{H}_{2}$ atmospheres that support liquid-water oceans and distinguish them from massive atmospheres (Figure 1, panel (b)). A companion paper has studied the atmospheric chemistry and spectral features of temperate planets with massive $\mathrm{H}_{2}$ atmospheres (Hu 2021), and now we turn to temperate planets with small $\mathrm{H}_{2}$ atmospheres. A recent paper might have similar intent as our work: Yu et al. (2021) studied the chemistry of temperate $\mathrm{H}_{2}$ atmospheres with varied surface pressures, with assumed zero flux for all species at the lower boundary. The theories of Yu et al. (2021) may thus be more applicable to arid rocky planets without substantial volcanic outgassing, and here we instead focus on ocean planets, and address how to identify them observationally. As we will show later, a small atmosphere on a temperate sub-Neptune will have a distinctive composition because of its interaction with the ocean underneath.

\section{Mutual Exclusivity of Habitability and Thermochemical Equilibrium}

On temperate sub-Neptunes, the condition to form a liquidwater ocean and that to achieve the thermochemical equilibrium of carbon and nitrogen molecules are mutually exclusive. The $\mathrm{CO}_{2}-\mathrm{CO}-\mathrm{CH}_{4}$ and $\mathrm{N}_{2}-\mathrm{NH}_{3}$ conversion rates are primarily a function of the temperature and to a lesser extent the pressure (Zahnle \& Marley 2014; Tsai et al. 2018), and in a temperate subNeptune like K2-18 b, the thermochemical equilibrium of carbon and nitrogen molecules are typically achieved at the pressure of $10^{7} \sim 10^{8} \mathrm{~Pa}$, where the temperature is $>1000 \mathrm{~K}$ (i.e., substantially higher than the critical point of water; Fortney et al. 2020; Hu 2021; Yu et al. 2021). Therefore, the gas-phase thermochemical equilibrium would be achieved in the deep and hot part of a massive atmosphere, and in contrast, it would not be achieved in a small atmosphere overlying a liquid-water ocean. Instead, $\mathrm{NH}_{3}$ and sulfur species would be sequestered by the ocean (Loftus et al. 2019, and also see Section 3) and the abundance of $\mathrm{CO}_{2}$ would be set by the ocean chemistry (Figure 2, with the cosmochemical and geological constraints detailed in the Appendix). This fundamental difference, coupled with atmospheric photochemistry, leads to distinctive gas abundances in the observable part $(<\sim 0.1 \mathrm{bar})$ of the atmosphere.

If the planet has a massive $\mathrm{H}_{2}$ atmosphere, thermochemical reactions in the deep atmosphere recycle $\mathrm{O}, \mathrm{C}, \mathrm{N}$, and $\mathrm{S}$ species into $\mathrm{H}_{2} \mathrm{O}, \mathrm{CH}_{4}, \mathrm{NH}_{3}$, and $\mathrm{H}_{2} \mathrm{~S}$ (Burrows \& Sharp 1999; Heng \& Tsai 2016; Blain et al. 2021; Woitke et al. 2021). $\mathrm{H}_{2} \mathrm{O}$ can form a cloud and the above-cloud $\mathrm{H}_{2} \mathrm{O}$ may be partially depleted as a result (Morley et al. 2014; Charnay et al. 2021; Hu 2021). Recent calculations have shown that the photodissociation of $\mathrm{NH}_{3}$ in the presence of $\mathrm{CH}_{4}$ leads to the formation of $\mathrm{HCN}$ and $\mathrm{N}_{2}$, and that $\mathrm{CO}$ and $\mathrm{CO}_{2}$ are produced by the photodissociation of $\mathrm{CH}_{4}$ together with $\mathrm{H}_{2} \mathrm{O}$ (Hu 2021). The photodissociation of $\mathrm{H}_{2} \mathrm{~S}$ leads to the formation of elemental sulfur haze (Hu et al. 2013; Zahnle et al. 2016), but the haze would likely be close to the cloud deck and would not mute transmission spectral features (Hu 2021). These photochemical products are transported to the deep atmosphere and recycled back to $\mathrm{CH}_{4}, \mathrm{NH}_{3}$, and $\mathrm{H}_{2} \mathrm{~S}$. An exception is that planets with super-solar atmospheric metallicity and appreciable internal heat may have additional $\mathrm{CO}, \mathrm{CO}_{2}$, and $\mathrm{N}_{2}$ transported from the deep troposphere and incomplete recycling to $\mathrm{NH}_{3}$ (Fortney et al. 2020; Hu 2021; Yu et al. 2021).

If the planet instead has a small atmosphere and a liquid-water ocean, the thermochemical recycling cannot occur. Instead, $\mathrm{CO}_{2}$ is the preferred form of carbon in equilibrium with a massive amount of $\mathrm{H}_{2} \mathrm{O}$ (Hu \& Seager 2014; Woitke et al. 2021), and $\mathrm{NH}_{3}$ is dissolved in the ocean and largely depleted from the atmosphere (see Section 3). The abundance of atmospheric $\mathrm{CO}_{2}$ is controlled by the oceanic pH (Kitzmann et al. 2015; Krissansen-Totton \& Catling 2017; Isson \& Planavsky 2018; Kite \& Ford 2018) and that of $\mathrm{N}_{2}$ is probably a combined result of the initial endowment and atmospheric escape. A reasonable lower bound of the total mass of $\mathrm{CO}_{2}$ in the $\mathrm{H}_{2}$ and $\mathrm{H}_{2} \mathrm{O}$ layers can be derived from the 


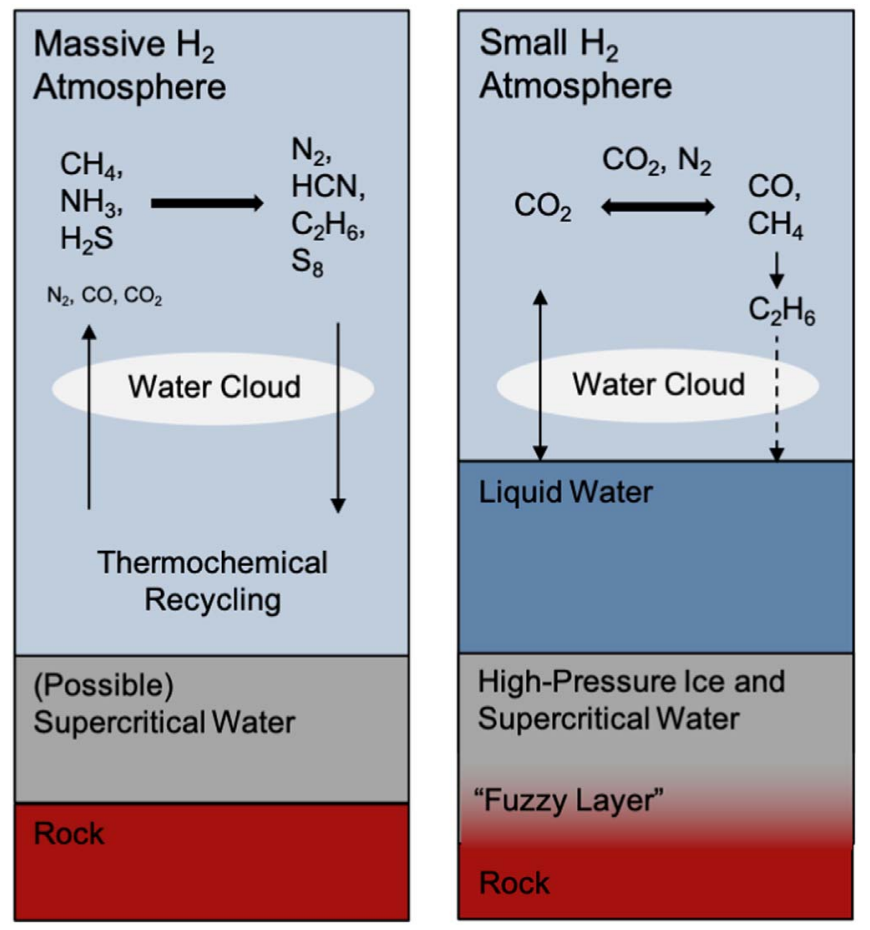

Figure 2. Interior structures of temperate H-rich exoplanets and the associated ranges of atmospheric composition. If the planet has a massive $\mathrm{H}_{2}$ atmosphere, the deep atmosphere would be hot-enabling thermochemical recycling-but a liquid-water surface would not be possible. If the planet has a small $\mathrm{H}_{2}$ atmosphere, a liquid-water surface may be possible. On these planets, the equilibrium abundance of atmospheric $\mathrm{CO}_{2}$ is set by the oceanic chemistry and that of $\mathrm{N}_{2}$ by atmospheric evolution.

cosmochemical constraints of planetary building blocks and the partitioning between the iron core, the silicate mantle, and the water layer (Appendix). Also, the "seafloor" of this thinatmosphere, $\mathrm{H}_{2} \mathrm{O}$-rich sub-Neptune will not be a sharp interface in density and composition, but instead have a finite thickness (Vazan et al. 2020). The interface will be compositionally stratified with denser material underlying less dense material, and material transport across this "fuzzy layer" is inhibited due to the stratification. Thus, any carbon or nitrogen added to the $\mathrm{H}_{2}$ and $\mathrm{H}_{2} \mathrm{O}$ envelope by planetesimal accretion late in planet growth will remain in the envelope, and will not be stirred down into the silicate layer. Meanwhile, transit observations can straightforwardly identify $\mathrm{H}_{2}$-dominated atmospheres and rule out $\mathrm{CO}_{2}$ - or $\mathrm{N}_{2}$-dominated ones only from the size of spectral features (MillerRicci et al. 2008).

One might also consider the intermediate situation between massive atmospheres with thermochemical equilibrium and small atmospheres with liquid-water oceans, e.g., the atmospheres with a surface pressure from a few to $\sim 100$ bars on K218 b. For many sub-Neptunes, this intermediate-atmosphere scenario would still require a massive water layer underneath to explain their mass and radius. If water was in the liquid form at the interface with the atmosphere, the evaporation of this ocean would make the atmosphere $\mathrm{H}_{2} \mathrm{O}$-dominated (Scheucher et al. 2020). If water is supercritical, any $\mathrm{H}_{2}$ layer of intermediate mass should be well mixed with the water layer. Therefore, such an intermediate endowment of $\mathrm{H}_{2}$ would most likely result in a non- $\mathrm{H}_{2}$-dominated atmosphere, which is, again, distinguishable with transmission spectroscopy (Miller-Ricci et al. 2008).

\section{Ocean Planet Models}

We have used an atmospheric photochemical model $(\mathrm{Hu}$ et al. 2012) coupled with a radiative-convective model (Scheucher et al. 2020) to determine the steady-state abundances of photochemical gases in small and temperate $\mathrm{H}_{2}$ atmospheres, for a cosmochemically and geologically plausible range of $\mathrm{CO}_{2}$ abundance, and compared the compositions and transmission spectra with the massive $\mathrm{H}_{2}$ atmosphere models published in $\mathrm{Hu}$ (2021). The massive-atmosphere models explored the atmospheric metallicity of $1-100 \times$ solar and included possible deep-tropospheric source $\mathrm{CO}, \mathrm{CO}_{2}$, and $\mathrm{N}_{2}$ and incomplete recycling of $\mathrm{NH}_{3}$ in super-solar atmospheres.

The photochemical model includes a comprehensive reaction network for $\mathrm{O}, \mathrm{H}, \mathrm{C}, \mathrm{N}$, and $\mathrm{S}$ species (including sulfur aerosols, hydrocarbons, and the reactions important in $\mathrm{H}_{2}$ atmospheres), and it has been used to study the lifetime and equilibrium abundance of potential biosignature gases in $\mathrm{H}_{2}$ atmospheres (Seager et al. 2013). We have updated the reaction network and tested the model with the measured photochemical gas abundance in the atmosphere of Jupiter (i.e., a lowtemperature $\mathrm{H}_{2}$ atmosphere; $\mathrm{Hu} 2021$ ).

The pressure-temperature profiles (Figure 3) used as the basis for the photochemical model are calculated with the climate module of 1D-TERRA (Scheucher et al. 2020). The module uses a correlated-k approach with the random overlap method to include molecular absorption, collision-induced opacities, and the continuum of water vapor to calculate the radiative equilibrium, and the appropriate (moist or dry) adiabatic lapse rate to apply the convection adjustment. The module has been tested against the cases of Earth, Venus, and Mars, as well as with other radiative-convective and 3D climate models for modeling steam atmospheres (Scheucher et al. 2020).

As examples, we study $\mathrm{H}_{2}$ atmospheres of 1 bar on a subNeptune planet that has a stellar irradiance similar to Earth and orbits around an early $\mathrm{M}$ star similar to K2-18. A 1 bar $\mathrm{H}_{2}$ atmosphere on such a planet would likely have a surface temperature consistent with a liquid-water ocean (Figure 3). We adopt the "ocean-planet" interpretation of the 1.7-3.5 $R_{\oplus}$ planet population that centers at $10 M_{\oplus}$, and $2.5 R_{\oplus}$ (Zeng et al. 2019; Venturini et al. 2020), and assume $50 \%$ of water by mass in this study. In this interpretation, sub-Neptunes may be ocean planets with deep oceans that do not require a massive $\mathrm{H}_{2}$ envelope to explain their radius, and can conceivably have moderate-size $\mathrm{H}_{2}$ atmospheres. This may not be directly applicable for $\mathrm{K} 2-18 \mathrm{~b}$, which resides on the low-density side of the $1.7-3.5 R_{\oplus}$ population. The specific choices of these parameters are, however, unimportant, because atmospheric chemistry is not sensitive to moderate changes in the surface gravity.

$\mathrm{CO}_{2}$ is the main form of carbon in thermochemical equilibrium with $\mathrm{H}_{2} \mathrm{O}(\mathrm{Hu} \&$ Seager 2014; Woitke et al. 2021). If a liquid-water ocean exists, the partial pressure of $\mathrm{CO}_{2}$ is set by atmosphere-ocean partitioning, which in turn is mainly controlled by the oceanic pH (Kitzmann et al. 2015; Krissansen-Totton \& Catling 2017; Isson \& Planavsky 2018; Kite \& Ford 2018). The $\mathrm{pH}$ is affected by the abundance of cations in the ocean, which come from complex water-rock reactions and dissolution of the seafloor. The rates of the processes involved are uncertain; therefore, we explore the mixing ratio of $\mathrm{CO}_{2}$ from $400 \mathrm{ppm}$ to $10 \%$, corresponding to the $\mathrm{pCO}_{2}$ range from the present-day Earth to early Earth 


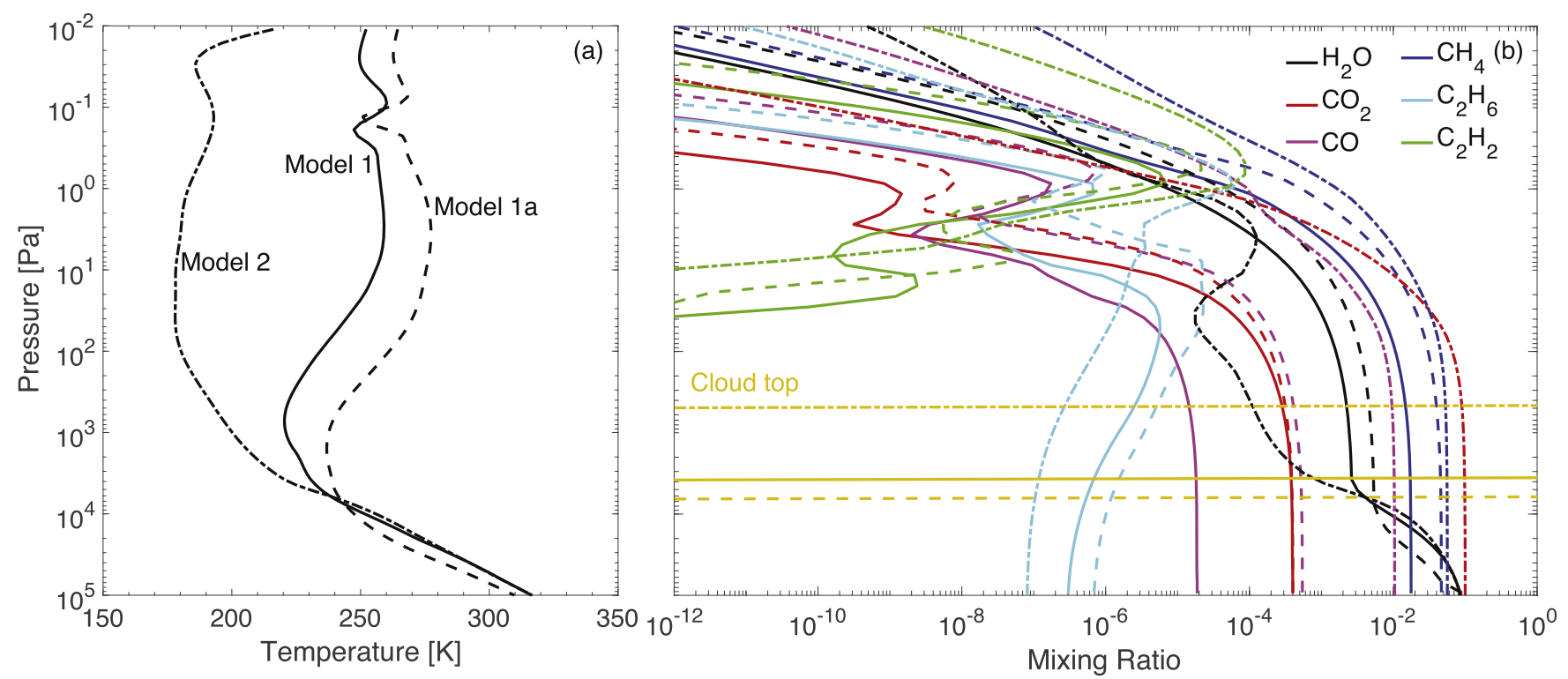

Figure 3. Modeled pressure-temperature profiles (a) and abundance profiles of main gases and photochemical products (b) in a temperate sub-Neptune like K2-18 b that has a small $\mathrm{H}_{2}$ atmosphere. Solid, dashed, and dashed-dotted lines show the results for the low- $\mathrm{CO}_{2}$ case (Model 1 in Table 1), the low-CO $\mathrm{CO}$ sources (Model 1a), and the high- $\mathrm{CO}_{2}$ case (Model 2). For the stellar irradiance, we use $S=S_{\text {Earth }} *\left(1-A_{\mathrm{B}}\right.$ ), with a Bond albedo of $A_{\mathrm{B}}=0.3$ (similar to Earth), to account for the radiative effects clouds would have in the otherwise cloud-free climate model. The surface albedo reflects a dark ocean (0.06). The surface temperatures in these models are consistent with a liquid-water ocean. The photochemical models use the UV spectrum of the M dwarf star GJ 176 (France et al. 2016; similar to K2-18; dos Santos et al. 2020). The steady-state mixing ratio $\mathrm{CH}_{4}$ is high and those of nitrogen molecules such as $\mathrm{NH}_{3}$ and $\mathrm{HCN}$ is $<10^{-12}$.

Table 1

Summary of the Photochemical Model Parameters and Results

\begin{tabular}{|c|c|c|c|c|c|c|c|}
\hline Model & Name & $\mathrm{CO}_{2}$ & CO Flux & $\mathrm{H}_{2} \mathrm{O}$ & $\mathrm{CO}$ & $\mathrm{CH}_{4}$ & $\mathrm{C}_{2} \mathrm{H}_{6}$ \\
\hline$\overline{1}$ & Low-CO $\mathrm{CO}_{2}$ & $4 \times 10^{-4}$ & 0 & $2.3 \times 10^{-3}$ & $1.4 \times 10^{-5}$ & $1.5 \times 10^{-2}$ & $3.0 \times 10^{-6}$ \\
\hline $1 \mathrm{a}$ & Low- $\mathrm{CO}_{2}$ Variant & $4 \times 10^{-4}$ & $1.0 \times 10^{9}$ & $3.3 \times 10^{-3}$ & $2.9 \times 10^{-4}$ & $2.9 \times 10^{-2}$ & $5.1 \times 10^{-6}$ \\
\hline 2 & High- $\mathrm{CO}_{2}$ & 0.1 & 0 & $1.1 \times 10^{-4}$ & $9.5 \times 10^{-3}$ & $5.3 \times 10^{-2}$ & $4.0 \times 10^{-7}$ \\
\hline
\end{tabular}

Note. The volume mixing ratio of $\mathrm{CO}_{2}$ (as inputs) is at the lower boundary, and those of $\mathrm{H}_{2} \mathrm{O}, \mathrm{CO}, \mathrm{CH}_{4}$, and $\mathrm{C}_{2} \mathrm{H}_{6}$ (as results) are column-averaged in $10-10^{3}$ Pa. The CO flux has a unit of $\mathrm{cm}^{-2} \mathrm{~s}^{-1}$.

(Catling \& Kasting 2017) and including the predicted range for ocean planets (Kite \& Ford 2018) that is still consistent with an $\mathrm{H}_{2}$-dominated atmosphere. The $4 \times 10^{-4}$ bar partial pressure of $\mathrm{CO}_{2}$ in the low- $\mathrm{CO}_{2}$ case, while not the absolute lower limit, is a cosmochemically and geologically reasonable lower bound of the $\mathrm{CO}_{2}$ partial pressure on an ocean planet (Appendix).

The mixing ratio of $\mathrm{N}_{2}$ on the modeled planet is probably set by atmospheric evolution (as opposed to the solubility equilibrium or geological recycling) and is assumed here to be $1 \%$. As $\mathrm{N}_{2}$ only minimally participates in the chemical cycles and does not have strong spectral features in the infrared, its exact abundance is not our main concern. The photochemical model indicates that the $\mathrm{NH}_{3}$ produced by photodissociation of $\mathrm{N}_{2}$ in $\mathrm{H}_{2}$ atmospheres has negligible mixing ratios $\left(<10^{-12}\right)$.

The pressure at the water-rock boundary of a $10-M_{\oplus}$ and $2.5-R_{\oplus}$ planet is $\sim 500 \mathrm{GPa}$ (Sotin et al. 2007; Levi et al. 2014), and this overloading pressure should suppress volcanism completely (Kite et al. 2009; Noack et al. 2017; Kite \& Ford 2018). Therefore we do not include any volcanic outgassing in the standard models. As variant models, we consider the possibility of minor and intermittent sources of $\mathrm{CO}$ into the atmosphere. Evaporation of meteorites may provide a source of $\mathrm{CO}$ and $\mathrm{CO}_{2}$ (Schaefer \& Fegley 2017), and waterrock reactions at the temperature relevant to the "fuzzy layer" may produce $\mathrm{CO}$ (and not $\mathrm{CH}_{4}$ as it is thermochemically disfavored at high temperatures). The rates of these processes are unknown, but numerical experiments with the photochemical model indicate that an additional $\mathrm{CO}$ source of $10^{10}$ molecule $\mathrm{cm}^{-2} \mathrm{~s}^{-1}$ would lead to a steady-state abundance of $\mathrm{CO}$ greater than that of $\mathrm{H}_{2}$, effectively resulting in a $\mathrm{CO}$ dominated atmosphere. A CO source of $10^{9}$ molecule $\mathrm{cm}^{-2} \mathrm{~s}^{-1}$ would produce the $\mathrm{CO}$-dominated atmosphere in the $10 \%-\mathrm{CO}_{2}$ case but not in the $400 \mathrm{ppm}-\mathrm{CO}_{2}$ case. We therefore include a low- $\mathrm{CO}_{2}$ case with the $\mathrm{CO}$ source of $10^{9}$ molecule $\mathrm{cm}^{-2} \mathrm{~s}^{-1}$ as a variant model.

Table 1 summarizes the input parameters and results of the photochemical models, and Figure 3 shows the profiles of temperature and mixing ratios of main gases and photochemical products. $\mathrm{CO}$ is produced from the photodissociation of $\mathrm{CO}_{2}$ and can build up to a $10^{-5}$ and $10^{-2}$ mixing ratio level for the low- $\mathrm{CO}_{2}$ and the high- $\mathrm{CO}_{2}$ cases. $\mathrm{OH}$ from the photodissociation of $\mathrm{H}_{2} \mathrm{O}$ destroys $\mathrm{CO}$ and maintains its steady-state mixing ratio. $\mathrm{CH}_{4}$ is also produced photochemically and can build up to a substantial mixing ratio $\left(10^{-3} \sim 10^{-2}\right)$. This effectiveness in producing $\mathrm{CH}_{4}$ from $\mathrm{CO}$ in temperate $\mathrm{H}_{2}$ atmospheres has also been noted in $\mathrm{Yu}$ et al. (2021). Together with the high $\mathrm{CH}_{4}$ mixing ratio, $\mathrm{C}_{2} \mathrm{H}_{6}$ is produced and can accumulate to a mixing ratio of $\sim 10^{-6} . \mathrm{C}_{2} \mathrm{H}_{2}$, as expected, is short-lived and only has significant mixing ratios in the upper 


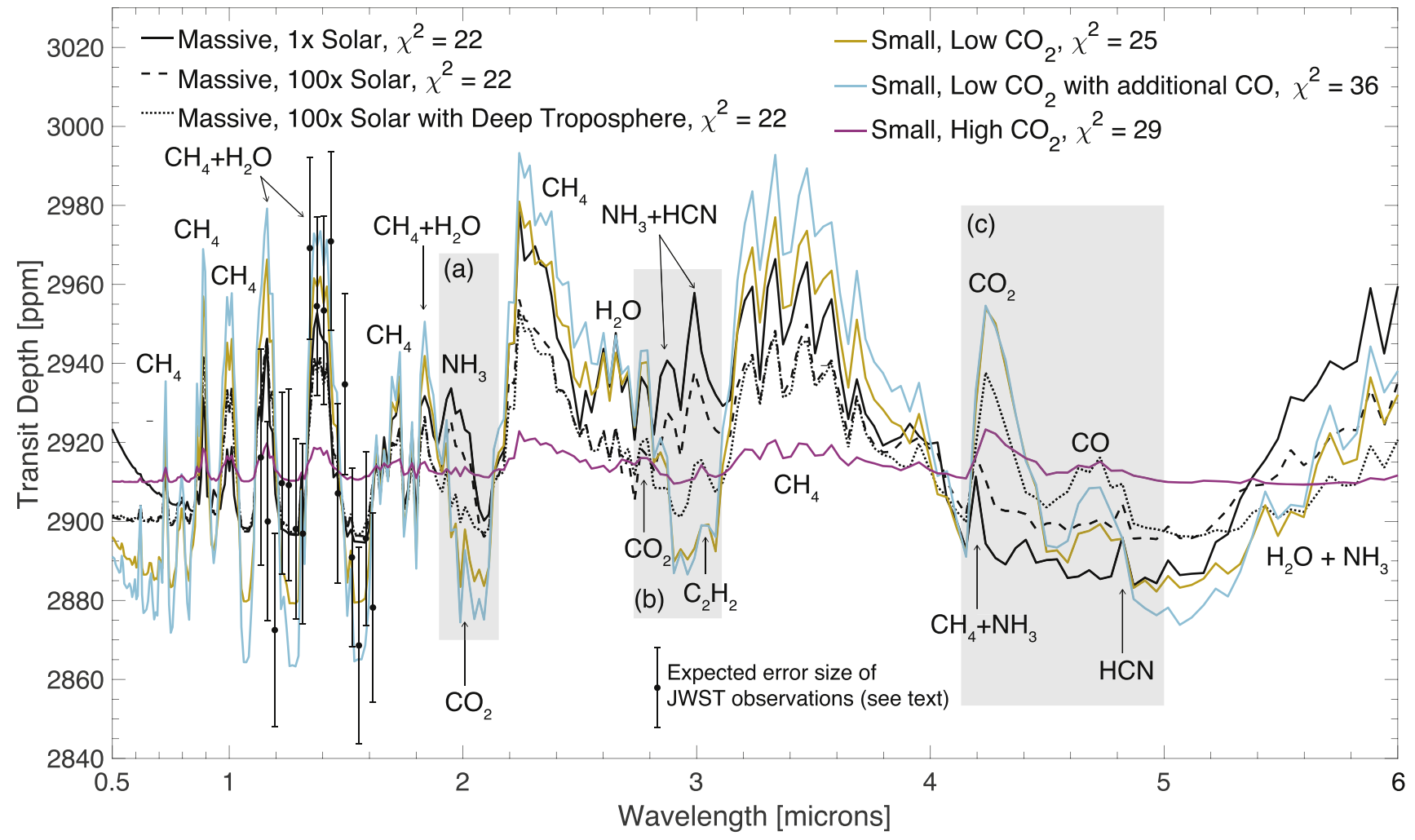

Figure 4. Modeled transmission spectrum of temperate sub-Neptune planets of $\mathrm{M}$ dwarf stars, using K2-18 $\mathrm{b}$ as an example and comparing with the planet's transit depth observed by Hubble (Benneke et al. 2019). The massive- $\mathrm{H}_{2}$-atmosphere models (black lines) and the small- $\mathrm{H}_{2}$-atmosphere models (colored lines) differ in three spectral regions: in (a) and (b), the massive-atmosphere models have absorption features of $\mathrm{NH}_{3}$ and $\mathrm{HCN}$, while the small-atmosphere models do not; in (c), the small-atmosphere models with a low mixing ratio of $\mathrm{CO}_{2}(400 \mathrm{ppm})$ have prominent features of $\mathrm{CO}_{2}$ and $\mathrm{CO}$, while the massive-atmosphere models only have small features of $\mathrm{NH}_{3}$ and $\mathrm{HCN}$. The $100 \times$ solar massive atmosphere with deep-tropospheric source and sink may have subdued $\mathrm{NH}_{3}$ and $\mathrm{HCN}_{\text {features and prominent } \mathrm{CO}_{2}}$ and $\mathrm{CO}$ features. The small-atmosphere models with a high mixing ratio of $\mathrm{CO}_{2}(10 \%)$ has a high mean molecular weight ( 6$)$ and a high cloud top (Figure 3$)$ and thus muted spectral features.

atmosphere. Here we have applied a deposition velocity of $10^{-5} \mathrm{~cm} \mathrm{~s}^{-1}$ for $\mathrm{C}_{2} \mathrm{H}_{6}$ to account for the loss of carbon due to organic haze formation and deposition (Hu et al. 2012); removing this sink does not substantially change the results shown in Figure 3. The additional source of $\mathrm{CO}$ would result in moderately more $\mathrm{CO}, \mathrm{CH}_{4}$, and $\mathrm{C}_{2} \mathrm{H}_{6}$ in the atmosphere (Model 1a in Table 1 and Figure 3). The photochemical CO and $\mathrm{CH}_{4}$ can build up to the mixing ratio levels that cause significant features in the planet's transmission spectrum (Section 4).

Before closing this section, we address whether $\mathrm{NH}_{3}$ can be produced substantially by water-rock reactions and then emitted into the atmosphere. Hydrothermal systems on early Earth may produce $\mathrm{NH}_{3}$ from the reduction of nitrite and nitrate (Summers \& Chang 1993; Summers 2005). On a planet with an $\mathrm{H}_{2}$-dominated atmosphere, however, atmospheric production of the oxidized nitrogen including nitrite and nitrate should be very limited. Moreover, the storage capability of $\mathrm{NH}_{3}$ by the ocean is vast and limits the emission into the atmosphere. At the $\mathrm{pH}$ value of 8 (a lower $\mathrm{pH}$ would further favor the partitioning of $\mathrm{NH}_{3}$ in the ocean), $10^{-6}$ bar of atmospheric $\mathrm{NH}_{3}$ requires a dissolved ammonium concentration of $10^{-3} \mathrm{moll}^{-1}$ in equilibrium (Seinfeld \& Pandis 2016). The mass of $\mathrm{NH}_{3}$ in the atmosphere and ocean is then $\sim 10^{-5}$ of the planetary mass. This would only be possible if much of the planet's rocky core begins with a volatile composition similar to carbonaceous chondrites, and most of this nitrogen is partitioned into the atmosphere and ocean as $\mathrm{NH}_{3}$ (Marty et al. 2016), which is highly unlikely as $\mathrm{N}_{2}$ is thermochemically favored. Therefore, the concentration of dissolved $\mathrm{NH}_{3}$ should be small and so is the atmospheric $\mathrm{NH}_{3}$ on a planet with a massive ocean.

\section{Spectral Characterization}

Figure 4 compares the expected spectra for the massiveatmosphere scenarios and the small-atmosphere scenarios. For K2-18 b the massive-atmosphere models with $1-100 \times$ solar metallicity and the small-atmosphere models with a low mixing ratio of $\mathrm{CO}_{2}$ (400 ppm) provide good fits to the transmission spectrum measured by Hubble.

Measuring the transmission spectra in an expanded wavelength range of $1-5 \mu \mathrm{m}$ will distinguish the small atmospheres from massive ones. Using K2-18 b as an example for temperate sub-Neptunes, we see that the massive-atmosphere models and the small-atmosphere models, while having differences within each group, can be distinguished using the spectral regions of 1.9-2.1, 2.7-3.1, and 4.1-5.0 $\mu \mathrm{m}$ (the shaded areas a, b, and c in Figure 4). Both the massive-atmosphere and small-atmosphere models show spectral features of $\mathrm{H}_{2} \mathrm{O}$ and $\mathrm{CH}_{4}$, and so observing these two gases alone is unlikely to separate the massive versus small scenarios.

At $1.9-2.1$ and $2.7-3.1 \mu \mathrm{m}$, the transmission spectra show $\mathrm{NH}_{3}$ and $\mathrm{HCN}$ absorption in massive atmospheres but not in small atmospheres. If the $100 \times$ solar massive atmosphere has incomplete $\mathrm{NH}_{3}$ recycling in the deep troposphere, it will have much weaker $\mathrm{NH}_{3}$ and $\mathrm{HCN}$ features in these spectral regions. The transmission spectra of small atmospheres show small $\mathrm{CO}_{2}$ 
features at $\sim 2.0$ and $\sim 2.75 \mu \mathrm{m}$, but the feature at $\sim 2.75 \mu \mathrm{m}$ is combined with a part of the $\mathrm{H}_{2} \mathrm{O}$ feature with similar strength. The transmission spectra of small atmospheres also show a small $\mathrm{C}_{2} \mathrm{H}_{2}$ feature at $\sim 3.05 \mu \mathrm{m}$, and given enough precision, it might be distinguishable with the $\mathrm{HCN}$ feature at $\sim 3.0 \mu \mathrm{m}$.

At $4.1-5.0 \mu \mathrm{m}$, the transmission spectra of small atmospheres (the low- $\mathrm{CO}_{2}$ cases) have prominent features of $\mathrm{CO}_{2}$ and $\mathrm{CO}$, while the spectra of massive atmospheres have weak features of $\mathrm{NH}_{3}$ and $\mathrm{HCN}$. If the $100 \times$ solar massive atmosphere has $\mathrm{CO}$ and $\mathrm{CO}_{2}$ transported from the deep troposphere, it can have prominent spectral features of $\mathrm{CO}_{2}$ and $\mathrm{CO}$ in this region as well.

From the above, we see that the $100 \times$ solar massive atmosphere with deep-tropospheric effects may resemble a small atmosphere in their transmission spectra (Figure 4), i.e., the lack of $\mathrm{NH}_{3}$ or $\mathrm{HCN}$ and the prominence of $\mathrm{CO}_{2}$ and $\mathrm{CO}$. Would this potential "false positive" be avoidable? The answer may be yes given enough precision and spectral resolution. First, the spectrum of the massive atmosphere with deeptropospheric effects still has weak spectral features of HCN, while none of the small atmospheres do. Second, the massive atmosphere has $\mathrm{CO}_{2} / \mathrm{CO}<\sim 0.1$, because $\mathrm{CO}$ always dominates over $\mathrm{CO}_{2}$ in the deep $\mathrm{H}_{2}$ troposphere of a temperate planet, and photochemical processes driven by an $\mathrm{M}$ dwarf star do not significantly raise the $\mathrm{CO}_{2}$ mixing ratio in the observable part of the atmosphere ( $\mathrm{Hu} 2021)$. In contrast, the small atmospheres typically have $\mathrm{CO}_{2} / \mathrm{CO} \geqslant 1$ (Table 1 ). In the more likely scenario without any volcanic outgassing, $\mathrm{CO}_{2} / \mathrm{CO} \sim 10$, because $\mathrm{CO}$ is produced photochemically from $\mathrm{CO}_{2}$. Therefore, by measuring the abundance of $\mathrm{CO}$ and $\mathrm{CO}_{2}$ independently, one could tell whether they are sourced from the deep troposphere.

Furthermore, a massive atmosphere with $\gg 100 \times$ solar metallicity will have a mean molecular weight much higher than that of an $\mathrm{H}_{2}$ atmosphere and is thus also distinguishable by transmission spectroscopy.

With moderate time investment (i.e., $<100 \mathrm{hr}$ ), JWST will provide the sensitivity to detect the signature gases aforementioned and distinguish massive versus small atmospheres on planets like K2-18 b. As an example, we have used PandExo (Batalha et al. 2017) to simulate the expected photometric precision using JWST's NIRSpec instrument. If combining two transit observations with NIRSpecs $\mathrm{G} 235 \mathrm{H}$ grating and four transits with the $\mathrm{G} 395 \mathrm{H}$ grating, the overall photometric precision would be $\sim 20$ ppm per spectral element at a resolution of $R=100$ in both channels that cover a wavelength range of $1.7-5.2 \mu \mathrm{m}$. These observations would distinguish the small-atmosphere scenarios versus the massive-atmosphere scenarios in Figure 4 with high confidence.

Additionally, we have performed spectral retrievals based on simulated observations using Tau-REx (Waldmann et al. 2015). We find that the mixing ratio of $\mathrm{NH}_{3}$ and $\mathrm{HCN}$ and the lack of $\mathrm{CO}_{2}$ or $\mathrm{CO}$ in the solar-abundance massive atmosphere would be usefully constrained (Figure 5). For the $100 \times$ solar atmosphere, the $\mathrm{CO}_{2}$ and $\mathrm{CO}$ transported from the deep troposphere would be identified, and the posteriors suggest that $\mathrm{CO}$ is likely more abundant than $\mathrm{CO}_{2}$. The reduction in the mixing ratios of $\mathrm{NH}_{3}$ and $\mathrm{HCN}$ due to incomplete recycling could also be seen in the retrieval, although the constraints on the mixing ratio of $\mathrm{HCN}$ is not accurate. For the small atmosphere, the retrieval yields degenerate solutions and thus double peaks in some posterior distributions. Despite this, it is clear from the posteriors that the atmosphere likely has high mixing ratios of both $\mathrm{CO}_{2}$ and $\mathrm{CH}_{4}$, has more $\mathrm{CO}_{2}$ than $\mathrm{CO}$, and has very little $\mathrm{NH}_{3}$ or $\mathrm{HCN}$ (Figure 5). In addition to JWST, the dedicated exoplanet atmosphere characterization mission ARIEL could also provide the sensitivity to detect these gases with more repeated transit observations (Changeat et al. 2020). This example shows that transit observations in the coming years can tell apart temperate sub-Neptunes with small $\mathrm{H}_{2}$ atmospheres versus the planets with massive atmospheres and reveal their distinct atmospheric composition.

\section{Discussion and Conclusion}

Taken together, the results presented above identify a near-term path to detect small $\mathrm{H}_{2}$ atmospheres that can be consistent with liquid-water oceans on temperate exoplanets. $\mathrm{H}_{2}$ atmospheres are probably the only type of temperate atmospheres readily within the reach of JWST and ARIEL for detailed studies, since characterizing a heavier $\mathrm{H}_{2} \mathrm{O}, \mathrm{N}_{2}$, or $\mathrm{CO}_{2}$ atmosphere will require coadding a few tens of transits-something not impossible but probably very hard (Belu et al. 2011; Krissansen-Totton et al. 2018; Wunderlich et al. 2019; Pidhorodetska et al. 2020; Gialluca et al. 2021). The mass of the $\mathrm{H}_{2}$ atmospheres - a parameter that is not directly measured by transits but critical for habitability if the planet is moderately irradiated — can be inferred from transmission spectra via the signature gases that indicate solubility equilibria versus gas-phase thermochemical recycling. The biggest uncertainty is probably the temperature at the $100 \sim 1000$ bar pressure level in the massive-atmosphere scenarios, which may be affected by heating mechanisms such as tidal heating. Detailed models of the interior temperature and mixing may further constrain this uncertainty (Fortney et al. 2020; Yu et al. 2021). Based on the range of the parameter space explored, we suggest that the sensitivity of multiple gases provided by future observatories' expanded wavelength coverage over Hubble would enable broad categorization of small versus massive atmospheres, summarized as a roadmap in Figure 1, panel (b).

How many sub-Neptunes could we expect to be ocean planets in the first place? The current population statistics of planets provide indirect evidence that most sub-Neptunes are not ocean planets (Owen \& Wu 2017; Fulton \& Petigura 2018; Jin \& Mordasini 2018), but most known planets are hotter than planets that can be habitable. Even if the current statistics apply to temperate planets, there is plenty of room to have $10 \%-20 \%$ of sub-Neptunes be ocean planets, which will still be a lot of planets. Also, some planets in or just below the "radius valley" may be sub-Neptunes that have evolved into ocean planets (Kite \& Schaefer 2021) and retained some residual $\mathrm{H}_{2}$ (Misener \& Schlichting 2021). For these reasons, this possibility of an ocean planet shrouded by a small $\mathrm{H}_{2}$ atmosphere should motivate detailed observations of temperate planets with radius from near the "radius valley" $\left(\sim 1.7 R_{\oplus}\right)$ to the main sub-Neptune population $\left(\sim 2.5 R_{\oplus}\right)$. If some of the temperate planets in the aforementioned group have small $\mathrm{H}_{2}$ atmospheres, their relative ease for transit observations would significantly enhance the prospect of detecting and characterizing potentially habitable exoplanets within the next decade.

The authors are thankful for helpful discussions with Fabrice Gaillard and Sukrit Ranjan. R.H. conceived and designed the study, simulated the photochemical models, interpreted the results, and wrote the manuscript. M.D. performed the JWST observation simulations and atmospheric retrievals. M.S. computed the pressure-temperature profiles. E.K. derived the 


\section{Massive atmosphere, $1 \times$ solar}

$\log \left(\mathrm{H}_{2} \mathrm{O}\right)=-5.30_{-3.13}^{+0.83} \log \left(\mathrm{CO}_{2}\right)=-10.33_{-1.15}^{+1.28} \log (\mathrm{CO})=-9.35_{-1.76}^{+1.90} \log \left(\mathrm{CH}_{4}\right)=-3.38_{-0.08}^{+0.08} \log \left(\mathrm{NH}_{3}\right)=-4.04_{-0.20}^{+0.17} \log (\mathrm{HCN})=-3.72_{-2.20}^{+0.72} \log \left(P_{\mathrm{clouds}}\right)=3.94_{-0.17}^{+0.14}$

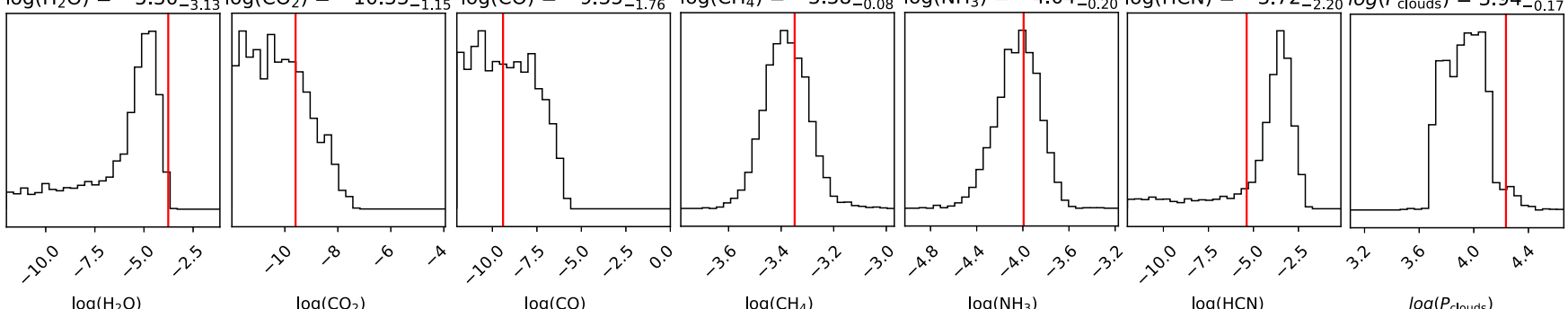

\section{Massive atmosphere, 100x solar with deep troposphere}

$\log \left(\mathrm{H}_{2} \mathrm{O}\right)=-5.22_{-4.23}^{+1.61} \log \left(\mathrm{CO}_{2}\right)=-4.66_{-0.52}^{+0.55} \log (\mathrm{CO})=-4.15_{-3.51}^{+0.89} \log \left(\mathrm{CH}_{4}\right)=-2.46_{-0.30}^{+0.42} \log \left(\mathrm{NH}_{3}\right)=-6.33_{-3.39}^{+1.57} \log (\mathrm{HCN})=-2.18_{-0.43}^{+0.19} \log \left(P_{\text {clouds }}\right)=3.12_{-0.26}^{+0.24}$

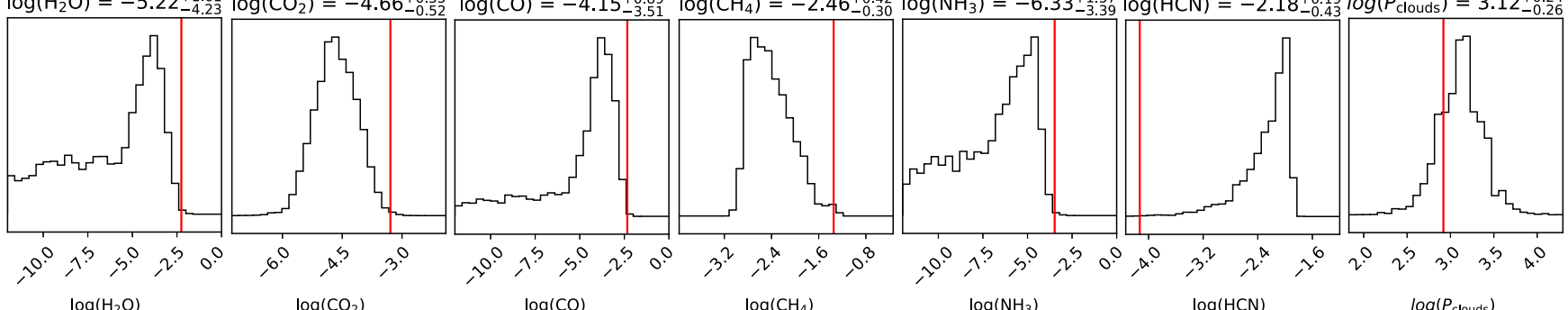

\section{Small atmosphere, low $\mathrm{CO}_{2}$}

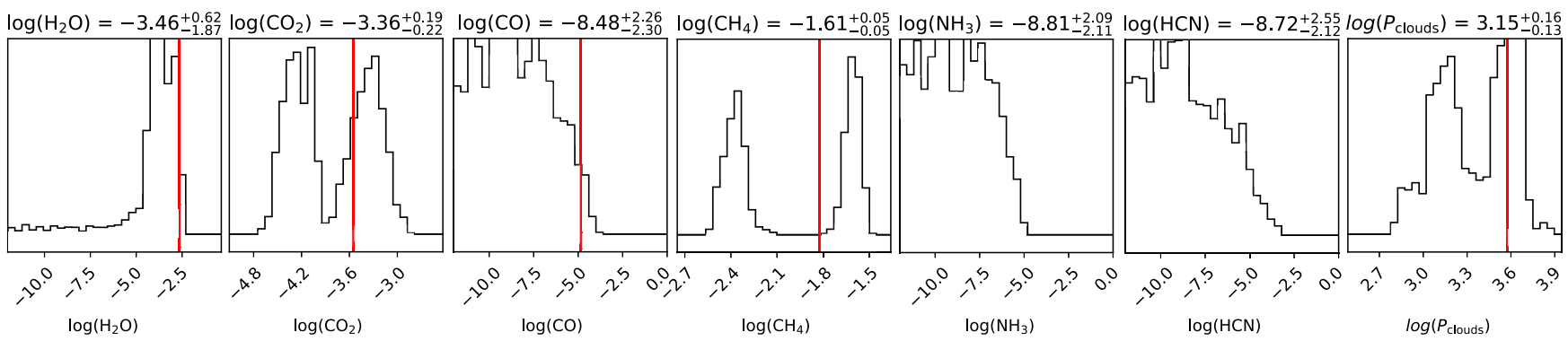

Figure 5. Retrieved posterior distributions of the abundances of the main chemical compounds and the cloud pressure in example massive-atmosphere and smallatmosphere scenarios. The input transmission spectra are calculated by Tau-REx using the atmospheric composition in Figure 3 and the expected uncertainties are calculated using PandExo (Batalha et al. 2017), assuming the combination of two transits of K2-18 b with NIRSpec/G235H and four transits with NIRSpec/G395H. The vertical red lines show the input value of the parameter, and the quantities on the top of each panel show the median and $1 \sigma$ values summarized from the posterior. The cloud pressure $\left(P_{\text {clouds }}\right)$ has a unit of Pa. A detailed characterization of the atmosphere of K2-18 b, including distinguishing a small atmosphere vs. a massive one and measuring the abundances of $\mathrm{H}_{2} \mathrm{O}, \mathrm{CH}_{4}, \mathrm{NH}_{3}, \mathrm{HCN}, \mathrm{CO}_{2}$, and $\mathrm{CO}$, will be achievable with moderate time investment of JWST.

cosmochemical and geological lower bounds for the carbon content. S.S. contributed interior structure models and insights. H.R. oversaw the development of the radiative-convective model used in the study. All authors commented on the overall narrative of the paper. The raw data that are used to generate the figures in this paper are available from the corresponding author upon reasonable request. This work was supported in part by NASA Exoplanets Research Program grant \#80NM0018F0612. The research was carried out at the Jet Propulsion Laboratory, California Institute of Technology, under a contract with the National Aeronautics and Space Administration.

\section{Appendix}

\section{Reasonable Lower Bound of $\mathrm{CO}_{2}$}

Is the $400 \mathrm{ppm} \mathrm{CO}_{2}$, or $4 \times 10^{-4}$ bar partial pressure in a 1 bar atmosphere, a reasonable lower bound of the $\mathrm{CO}_{2}$ partial pressure on an ocean planet? We consider this question from a cosmochemical and geochemical perspective. Assuming equilibrium (during planet formation) between a Fe-core, a silicate mantle, and a well-mixed supercritical volatile envelope, the partitioning of $\mathrm{C}$ mass between reservoirs is described by

$$
\mathrm{C}_{\text {total }}=\mathrm{C}_{\text {core }}+\mathrm{C}_{\text {silicate }}+\mathrm{C}_{\text {envelope }},
$$

where all reservoir masses are in kilograms, and

$$
\mathrm{C}_{\text {core }} / M_{\text {core }}=D_{\mathrm{C}}\left(\mathrm{C}_{\text {silicate }} / M_{\text {silicate }}\right) \text {, }
$$

where $D_{\mathrm{C}}$ is a dimensionless partition coefficient, $M_{\text {core }}(\mathrm{kg})$ is the mass of the Fe-dominated core, and $M_{\text {silicate }}(\mathrm{kg})$ is the mass of the silicate mantle (molten during planet formation). For the partitioning between the envelope and the silicate mantle,

$$
\mathrm{C}_{\text {envelope }} k\left(g_{\text {esi }} / A_{\text {esi }}\right)\left(\mu_{\text {avg }} / \mu_{\mathrm{C}}\right) s_{\mathrm{C}}=\mathrm{C}_{\text {silicate }} / M_{\text {silicate }},
$$

where $k$ is a stochiometric correction from $\mathrm{C}$ mass to the mass of the C-bearing species in the envelope (i.e., 44/12 3.7 for $\left.\mathrm{CO}_{2}\right), g_{\text {esi }}$ is gravitational acceleration at the envelope-silicate interface in $\mathrm{m} \mathrm{s}^{-2}, A_{\mathrm{esi}}$ is the area of the envelope-silicate interface in $\mathrm{m}^{2}, \mu_{\text {avg }}$ is the average molecular weight of the envelope (in Da), $\mu_{\mathrm{C}}$ is the molecular weight of the $\mathrm{C}$-bearing 
species (in Da), and $s_{\mathrm{C}}$ is the solubility of the C-bearing species (in $\mathrm{Pa}^{-1}$ ). Here we have assumed that the molten silicate layer is well-stirred.

Supposing $M_{\text {core }} / M_{\text {silicate }} \sim 0.5$ (like Earth) and $D_{\mathrm{C}} \sim 10^{3}$ (Dasgupta 2013), then $\mathrm{C}_{\text {core }} / \mathrm{C}_{\text {silicate }} \sim 500$. If $\mathrm{C}_{\text {silicate }} / M_{\text {silicate }} \sim$ $50 \mathrm{ppm}$ then $\mathrm{C}_{\text {core }} / M_{\text {core }} \sim 2.5 \mathrm{wt} \%$, or $\mathrm{C}_{\text {total }} /\left(M_{\text {core }}+M_{\text {silicate }}\right) \sim$ $1 \mathrm{wt} \%$, which is a reasonable lower bound for the primordial carbon endowment (see below). For $s_{\mathrm{C}}=0.55 \mathrm{ppm} \mathrm{Mpa}^{-1}$ (Dasgupta \& Grewal 2019), the envelope partial pressure of the C species $\left(=\mathrm{C}_{\text {envelope }} k\left(g_{\text {esi }} / A_{\text {esi }}\right)\left(\mu_{\text {avg }} / \mu_{\mathrm{C}}\right)\right)$ is $\sim 10^{3}$ bars. For a $5-M_{\oplus}$ and $1.5-R_{\oplus}$ core + mantle (Zeng et al. 2019) that defines the envelope-silicate boundary, and $\mu_{\mathrm{avg}} / \mu_{\mathrm{C}}=0.4$ (appropriate for $\mathrm{CO}_{2}$ in a $\mathrm{H}_{2} \mathrm{O}$-dominated supercritical layer during planet formation), the $\mathrm{CO}_{2}$ mass in the envelope is $0.2 \%$ of an Earth mass. This estimate shows that even though most $\mathrm{C}$ is in the core, still-significant reservoirs of $\mathrm{C}$ exist both in the silicate and in the envelope (Bergin et al. 2015; Hirschmann 2016; Dasgupta \& Grewal 2019; Keppler \& Golabek 2019). Recent indications that the partition coefficient $D_{\mathrm{C}}$ is $\ll 10^{3}$ at the pressures and temperatures that are relevant for assembly of sub-Neptunes (Fischer et al. 2020) would imply even more envelope C enrichment.

Following the formation of the liquid-water ocean, almost all of the $\mathrm{CO}_{2}$ will be dissolved in the ocean. For a $5-M_{\oplus}$ water layer, the $\mathrm{CO}_{2}$ mass in the envelope estimated above corresponds to a concentration of $\sim 0.01 \mathrm{~mol} \mathrm{l}^{-1}$ of dissolved $\mathrm{CO}_{2}$. Here we have also assumed that the ocean is well-stirred. A higher oceanic $\mathrm{pH}$ leads to more effective dissolution and less $\mathrm{CO}_{2}$ in the atmosphere. As an extreme, if cations are leached from the silicate and not charge-balanced by chloride ions, then an ocean composition with a $\mathrm{pH}$ of 9-10 ("a soda lake") will result (Kempe \& Degens 1985). Using the equilibrium constant of carbonate and bicarbonate dissociation (Seinfeld \& Pandis 2016), the $\mathrm{CO}_{2}$ partial pressure in equilibrium with this ocean would be $5 \times 10^{-5} \sim 7 \times 10^{-4}$ bar, which is consistent with the assumed lower bound.

The partition coefficient gives the ratios of concentration of a species in the Fe-dominated core to the concentration of the same species in the silicate mantle. Therefore doubling the total amount of $\mathrm{C}$ in the core+mantle will double the concentration in the magma. What is the whole-planet $C$ content? In principle, a planet can form without accreting volatiles. However, a thin-atmosphere sub-Neptune must have a thick volatile $\left(\mathrm{H}_{2} \mathrm{O}\right)$ layer in order to match density data. It is very likely that a world that forms with tens of wt $\% \mathrm{H}_{2} \mathrm{O}$ will also accrete abundant $\mathrm{C}$. We develop this point in more detail in the following paragraph.

At $T_{\text {eff }} \sim 300 \mathrm{~K}$, the minimum liquid-water content to explain most sub-Neptune masses and radii is $>\sim 50 \mathrm{wt} \%$ even if there is no Fe-metal core (Mousis et al. 2020). This is more $\mathrm{H}_{2} \mathrm{O}$ than can possibly be produced by hydrogen-magma reactions (Kite \& Schaefer 2021), and instead implies a contribution of planet building blocks from the temperature range beyond the water ice snowline. This is a zone where (in the solar system) abundant refractory carbon is found. Specifically, the carbon content of primitive chondrite meteorites (CI and CM type) is 2-6 wt\% (Pearson et al. 2006). Although we do not fully understand the origin of this refractory carbon, proposed mechanisms for forming this refractory carbon would also apply to exoplanetary systems (Bergin et al. 2014). Therefore we assume a planet bulk composition of $(2-6 \mathrm{wt} \%) \times(1-x)$ carbon, where $x$ is the $\mathrm{H}_{2} \mathrm{O}$ mass fraction, and the remainder of the planet's building blocks are assumed to have $\mathrm{C}$ content similar to that of primitive chondrites. This is a conservative lower limit on bulk $\mathrm{C}$ content for a thin- $\mathrm{H}_{2}$-atmosphere sub-Neptune, for the following two reasons. (i) It considers only refractory $\mathrm{C}$, not $\mathrm{C}$ ices (e.g., $\mathrm{CO}_{2}$ ice), which could be important in the case of whole-planet migration. (ii) Some primitive bodies in the solar system appear to be more $\mathrm{C}$-rich than the most primitive chondrite meteorites; for example, the surface of the dwarf planet Ceres may contain 20 wt\% C (Marchi et al. 2019). These large bulk C contents map to substantial envelope $\mathrm{C}$ contents (Equations (A1)-(A3)). As such, the $4 \times 10^{-4}$ bar partial pressure of $\mathrm{CO}_{2}$, while not the absolute lower limit, is a cosmochemically and geologically reasonable lower bound of the $\mathrm{CO}_{2}$ partial pressure on an ocean planet.

\section{ORCID iDs}

Renyu Hu (iD https://orcid.org/0000-0003-2215-8485 Mario Damiano (D) https://orcid.org/0000-0002-1830-8260 Markus Scheucher (iD https://orcid.org/0000-0003-4331-2277 Edwin Kite (iD https://orcid.org/0000-0002-1426-1186 Sara Seager (iD https://orcid.org/0000-0002-6892-6948 Heike Rauer (iD https://orcid.org/0000-0002-6510-1828

\section{References}

Batalha, N. E., Mandell, A., Pontoppidan, K., et al. 2017, PASP, 129, 064501 Belu, A., Selsis, F., Morales, J.-C., et al. 2011, A\&A, 525, A83 Benneke, B., Wong, I., Piaulet, C., et al. 2019, ApJL, 887, L14 Bergin, E., Cleeves, L. I., Crockett, N., \& Blake, G. 2014, FaDi, 168, 61 Bergin, E. A., Blake, G. A., Ciesla, F., Hirschmann, M. M., \& Li, J. 2015, PNAS, 112, 8965

Blain, D., Charnay, B., \& Bézard, B. 2021, A\&A, 646, A15

Burrows, A., \& Sharp, C. 1999, ApJ, 512, 843

Catling, D. C., \& Kasting, J. F. 2017, Atmospheric Evolution on Inhabited and Lifeless Worlds (Cambridge: Cambridge Univ. Press)

Changeat, Q., Edwards, B., Al-Refaie, A. F., et al. 2020, arXiv:2003.01486

Charnay, B., Blain, D., Bézard, B., et al. 2021, A\&A, 646, A171

Cowan, N. B., Agol, E., Meadows, V. S., et al. 2009, ApJ, 700, 915

Dasgupta, R. 2013, RvMG, 75, 183

Dasgupta, R., \& Grewal, D. S. 2019, in Deep Carbon: Past to Present, ed

B. Orcutt, I. Daniel, \& R. Dasgupta (Cambridge: Cambridge Univ. Press), 4 dos Santos, L. A., Ehrenreich, D., Bourrier, V., et al. 2020, A\&A, 634, L4

Fan, S., Li, C., Li, J.-Z., et al. 2019, ApJL, 882, L1

Fischer, R. A., Cottrell, E., Hauri, E., Lee, K. K., \& Le Voyer, M. 2020, PNAS, 117,8743

Fortney, J. J., Visscher, C., Marley, M. S., et al. 2020, AJ, 160, 288

France, K., Loyd, R. P., Youngblood, A., et al. 2016, ApJ, 820, 89

Fulton, B. J., \& Petigura, E. A. 2018, AJ, 156, 264

Gaudi, B. S., Seager, S., Mennesson, B., et al. 2020, arXiv:2001.06683

Gialluca, M. T., Robinson, T. D., Rugheimer, S., \& Wunderlich, F. 2021, PASP, 133, 054401

Heng, K., \& Tsai, S.-M. 2016, ApJ, 829, 104

Hirschmann, M. M. 2016, AmMin, 101, 540

Hu, R. 2021, ApJ, 921, 27

Hu, R., \& Seager, S. 2014, ApJ, 784, 63

Hu, R., Seager, S., \& Bains, W. 2012, ApJ, 761, 166

Hu, R., Seager, S., \& Bains, W. 2013, ApJ, 769, 6

Isson, T. T., \& Planavsky, N. J. 2018, Natur, 560, 471

Jin, S., \& Mordasini, C. 2018, ApJ, 853, 163

Kempe, S., \& Degens, E. T. 1985, ChGeo, 53, 95

Keppler, H., \& Golabek, G. 2019, Geochem. Perspect. Lett., 11, 12

Kite, E. S., \& Ford, E. B. 2018, ApJ, 864, 75

Kite, E. S., Manga, M., \& Gaidos, E. 2009, ApJ, 700, 1732

Kite, E. S., \& Schaefer, L. 2021, ApJL, 909, L22

Kitzmann, D., Alibert, Y., Godolt, M., et al. 2015, MNRAS, 452, 3752

Koll, D. D., \& Cronin, T. W. 2019, ApJ, 881, 120

Krissansen-Totton, J., \& Catling, D. C. 2017, NatCo, 8, 15423

Krissansen-Totton, J., Garland, R., Irwin, P., \& Catling, D. C. 2018, AJ, 156,114

Lee, E. J., \& Chiang, E. 2016, ApJ, 817, 90

Levi, A., Sasselov, D., \& Podolak, M. 2014, ApJ, 792, 125

Loftus, K., Wordsworth, R. D., \& Morley, C. V. 2019, ApJ, 887, 231 
Madhusudhan, N., Nixon, M. C., Welbanks, L., Piette, A. A., \& Booth, R. A. 2020, ApJL, 891, L7

Marchi, S., Raponi, A., Prettyman, T., et al. 2019, NatAs, 3, 140

Marty, B., Avice, G., Sano, Y., et al. 2016, E\&PSL, 441, 91

Miller-Ricci, E., Seager, S., \& Sasselov, D. 2008, ApJ, 690, 1056

Misener, W., \& Schlichting, H. E. 2021, MNRAS, 503, 5658

Morley, C. V., Marley, M. S., Fortney, J. J., et al. 2014, ApJ, 787, 78

Mousis, O., Deleuil, M., Aguichine, A., et al. 2020, ApJL, 896, L22

Nixon, M. C., \& Madhusudhan, N. 2021, MNRAS, 505, 3414

Noack, L., Rivoldini, A., \& Van Hoolst, T. 2017, PEPI, 269, 40

Owen, J. E., \& Wu, Y. 2017, ApJ, 847, 29

Pearson, V., Sephton, M., Franchi, I., Gibson, J., \& Gilmour, I. 2006, M\&PS, 41, 1899

Pidhorodetska, D., Fauchez, T. J., Villanueva, G. L., Domagal-Goldman, S. D., \& Kopparapu, R. K. 2020, ApJL, 898, L33

Pierrehumbert, R., \& Gaidos, E. 2011, ApJL, 734, L13

Ramirez, R. M., \& Kaltenegger, L. 2017, ApJL, 837, L4

Robinson, T. D., Meadows, V. S., \& Crisp, D. 2010, ApJL, 721, L67

Rogers, L., \& Seager, S. 2010, ApJ, 716, 1208

Schaefer, L., \& Fegley, B. 2017, ApJ, 843, 120

Scheucher, M., Wunderlich, F., Grenfell, J. L., et al. 2020, ApJ, 898, 44
Seager, S., Bains, W., \& Hu, R. 2013, ApJ, 777, 95

Seinfeld, J. H., \& Pandis, S. N. 2016, Atmospheric Chemistry and Physics: From Air Pollution to Climate Change (New York: Wiley)

Sotin, C., Grasset, O., \& Mocquet, A. 2007, Icar, 191, 337

Summers, D. P. 2005, OLEB, 35, 299

Summers, D. P., \& Chang, S. 1993, Natur, 365, 630

Tsai, S.-M., Kitzmann, D., Lyons, J. R., et al. 2018, ApJ, 862, 31

Tsiaras, A., Waldmann, I. P., Tinetti, G., Tennyson, J., \& Yurchenko, S. N. 2019, NatAs, 3, 1086

Valencia, D., Guillot, T., Parmentier, V., \& Freedman, R. S. 2013, ApJ, 775,10

Vazan, A., Sari, R., \& Kessel, R. 2020, arXiv:2011.00602

Venturini, J. E., Guilera, O. M., Haldemann, J., Ronco, M. P., \& Mordasini, C. 2020, A\&A, 643, L1

Waldmann, I. P., Tinetti, G., Rocchetto, M., et al. 2015, ApJ, 802, 107

Woitke, P., Herbort, O., Helling, C., et al. 2021, A\&A, 646, A43

Wunderlich, F., Godolt, M., Grenfell, J. L., et al. 2019, A\&A, 624, A49

Yu, X., Moses, J. I., Fortney, J. J., \& Zhang, X. 2021, ApJ, 914, 38

Zahnle, K., Marley, M. S., Morley, C. V., \& Moses, J. I. 2016, ApJ, 824, 137

Zahnle, K. J., \& Marley, M. S. 2014, ApJ, 797, 41

Zeng, L., Jacobsen, S. B., Sasselov, D. D., et al. 2019, PNAS, 116, 9723 